\title{
An Heterogeneous wireless networks testbed for Smart Environment scenarios
}

\author{
S. Melzi, S. Napoli, A. Pollastro and Scott Fowler
}

\section{Linköping University Post Print}

\section{Tweet}

N.B.: When citing this work, cite the original article.

(C2014 IEEE. Personal use of this material is permitted. However, permission to reprint/republish this material for advertising or promotional purposes or for creating new collective works for resale or redistribution to servers or lists, or to reuse any copyrighted component of this work in other works must be obtained from the IEEE.

S. Melzi, S. Napoli, A. Pollastro and Scott Fowler, An Heterogeneous wireless networks testbed for Smart Environment scenarios, 2014, 2014 IEEE 19th International Workshop on Computer Aided Modeling and Design of Communication Links and Networks (CAMAD), 173-177. http://dx.doi.org/10.1109/CAMAD.2014.7033229

Postprint available at: Linköping University Electronic Press http://urn.kb.se/resolve?urn=urn:nbn:se:liu:diva-117751 


\title{
An Heterogeneous wireless networks testbed for Smart Environment scenarios
}

\author{
Stefano Melzi*, Stefano Napoli*, Alberto Pollastro*, Scott Fowler ${ }^{\ddagger}$ \\ ${ }^{*}$ MobiMESH s.r.l., Milan, Italy \\ ${ }^{\ddagger}$ Mobile Telecoms., Department of Science \& Technology, Linköping University, Norrköping, Sweden
}

\begin{abstract}
Smart Environments are environments where heterogeneous wireless networks are implemented and connected together both in terms of networking and in terms of functionalities, thus providing an abstraction layer to higher level applications that can exploit the interplay of such technologies to obtain killer features. In order to obtain real advantages from the integration of heterogeneous components the scenarios shall be clearly understood and analysed;therefore this paper classifies the different scenarios and network architectures that can be employed in creating a Smart Environment. Moreover, real life experimenting is needed, through testbeds and trial installations; our testbed is therefore described, as well as some results on the effects achieved by interconnecting different technologies.
\end{abstract}

\section{INTRODUCTION}

The tremendous developments happened in both technologies and infrastructures in the last years have led to a wide range of applications which have a deep impact on people's life. Applications exploit wirless technologies to communicate with objects and devices in order to have the environment adapt to its inhabitants; such adaptive environments can be defined Smart Environments. The concept of Smart Environment is very broad, since it ranges from small scale scenarios such as Smart Offices to metro-wide scenarios called Smart Cities; all of them shall be characterized by a pervasive networking infrastructure with wireless access and a high degree of interaction between the different technologies. The challenge for Smart Environments is therefore in the interaction of the different wireless technologies and in the integration that can be achieved between the components, thus hiding the underlying complexity to the end users. In this paper we provide a description of two major Smart Environment scenarios, and of the general architecture of the networks that build up the infrastructure of the Smart Environments; then we briefly cover the different technologies that are employed to create such networks, and finally we describe the testbed which we have built to experiment Smart Environment applications.

\section{SCENARIOS}

Smart Environment scenarios may be very different from each other, depending on the size, the type, the main end users, etc. In this section we describe two scenarios that can be considered representative for the major categories, since they coverthe large scale, outdoor scenario which is usually called Smart City, and the smaller scale, indoor scenario that can be called Smart Office.

\section{A. Smart City}

The Smart City scenario [1] considers a city which owns a pervasive, multi-service network which is employed by the different service providers and by the users to simplify their living. The major topics that can be covered by such a network are public safety applications (video surveillance, emergency calls, people flow analysis, etc...), infomobility (public transportation information, traffic management, timesheets, etc...), WiFi HotSpots, localized services (tourist information, parking information, etc...), sensor networks (pollution detection, energy saving applications, etc...), smart metering applications. The latter in particular is an interesting emerging field since on one side the regulatory Authorities are forcing the utilities to deploy smart metering networks in order to efficiently control and monitor end users' devices and in order to provide smart network-wide policies. On the other side, the only way of having a cost-effective and sustainable smart metering network for an utility is to share costs and capacity with other entities on the territory.

\section{B. Smart Office}

The Smart Office scenario covers an indoor environment in which a network is deployed in order to provide services and applications to its inhabitants; in the office case such services can be targeted onto improving the productivity of the employees (with applications that fasten some procedures and that foster the adoption of good working practices), improving the security (with 
applications that connect alarm systems, presence detection and people localization, and with ICT security applications) and reducing the operative costs (for example with energy saving applications, heating/cooling control, telephone controls, etc).

\section{NETWORK ARCHITECTURE}

The network architecture needed for a Smart Environment network can be summarized in several components, which may vary in terms of technology and of deployment, but which can be abstracted for a general view. The general architecture can be considered a wireless heterogeneous mesh network, since it usually involves a possibly multi-hop backbone network, one or more wireless access networks, several sensor and metering extensions, and localization systems. These components are briefly described in the following sections.

\section{A. Backbone}

The backbone is the main transportation network, whose main tasks are backhauling through the territory and gathering data from the different access and extension networks and transporting them to the Internet access(es). The backbone is usually created with broadband technologies, and it can be made by a mix of wired and wireless technologies. It can adopt several network topologies, even though ring-oriented topologies tend to be preferred since they grand better protection.

\section{B. Access Network}

The access network is a component of the architecture whose role is enabling the connection of end users' devices. Examples of access networks are the WiFi HotSpot, the Smart Metering access network for connecting the user's counters, the Hiperlan $5 \mathrm{GHz}$ links made to connect wireless videosurveillance cameras, and so on.

\section{Extension networks}

Sevreal extension networks can be deployed in an heterogeneous Smart Environment scenario in order to connect vertical application-driven networks to the main, shared backbone. The integration of such extension networks with the backbone is usually achieved through gateways, that allow the connection of the two network branches by being equipped with different interfaces. Some examples of extension networks are sensor networks, metering networks, PLC networks and so on.

\section{Localization}

Localization is a key aspect in a Smart Environment network, since it can provide information that can be helpful for every application. Localization can be achieved in several ways, both through the existing infrastructure and through separate and additional networks. When localization is made through the existing infrastructure, it is usually achieved through software components that run measurements on the signals and that are capable of performing the necessary calculations; it is the case of WiFi based localization, which can be carried out over the WiFi Access network.

Other localization solutions rely on separate networks; it is the case of GPS, very useful and precise in outdoor environment, and the case of Bluetooth Low Energy (BLE) based localization, which is less precise but still effective in some applications.

\section{Smart EnVironment Technologies}

The above mentioned architectures can be built by employing heterogeneous technologies, interconnected through routers and gateways. The following paragraphs describe the technologies which are employed in the most common Smart Environment scenarios.

\section{A. WiFi}

$\mathrm{WiFi}$ is the speaking name for the IEEE 802.11 goup of specifications related to wireless transmission technology for IP networks [2]. It works in the ISM $2.4 \mathrm{GHz}$ band with a maximum transfer data rate up to $300 \mathrm{Mbps}$ (IEEE 802.11n). It is a very pervasive and widespread technology thanks to its simplicity and low costs, and to the use of unlicensed bands. The new IEEE 802.11ac standard [3] avoids radio congestion by extending the usage to the $5 \mathrm{GHz}$ radio band, thus being able to reach data rates up to $1,69 \mathrm{~Gb} / \mathrm{s}$. Today $\mathrm{WiFi}$ is used to provide ubiquitous internet access thanks to the great diffusion of WiFi interfaces on most end-user devices and machines.

\section{B. Wireless Metering Bus}

Many solutions have been proposed for the metering applications [4]; however the Open Metering System group in Europe reccomends the adoption of the Wireless Metering Bus protocol (WMBus) [5]. The first version of the WMBus employs the $868 \mathrm{MHz}$ ISM and $468 \mathrm{MHz}$ bands, while the following versions (EN 13757-4:2011) extended the range to the $169 \mathrm{MHz}$ band. Lower frequency bands, in fact, enable longer trasmission ranges, which are necessary to connect the indoor meters with the outdoor readers, while the very low data rates allow ultra low power operations for battery 
powered devices [6]. The interconnection between a WMBus network and a WiFi network can be done with dual interface gateways that can collect data sent by water, gas and heating meters and forward it to their specific utility. The major advantage with respect to M2M communication through the GSM network is that WMBus power requirement are lower than GSM and meter equipment can be powered with battery, solar or other alternative sources [7].

\section{Zigbee}

Zigbee [8] is a protocol specification used to create low power wireless network based on the IEEE 802.15.4 standard [9]. The IEEE 802.15.4 covers PHY and MAC layers for embedded devices operating in the ISM band with a data rate limited to $250 \mathrm{kbps}$ and with low power requirements. Zigbee defines a network layer on top of the IEEE 802.15.4 stack, in order to create multi-hop clusters of devices with different topologies (star, tree and peer-to-peer). A Zigbee network is composed of devices with different roles:

- Full Function Device (FFD): devices with greater computation and memory capabilities that can perform packet routing. Every network must feature at least be one FDD that acts as PAN Coordinator to administer network operation.

- Reduced Function Device (RFD): simple devices that can only connect to a network without routing capabilities.

Zigbee also defines different profiles (Home Automation, Smart metering, Personal Home and Hospital Care) which determine the packet payloads and the communication for many kind of devices (lighting controller, infrared sensors, temperature sensors), in order to allow equipments from different manufacturers to interoperate. The Zigbee standard was developed to create a wireless network that can connect smart sensors with a coverage area of some hundred meters; that is the reason why it is usually employed as a technology for the extension network, thus acting locally and delivering the data to the backbone for long range distribution.

\section{Bluetooth Low Energy}

Bluetooth Low Energy (BLE) [10] [11] was introduced as part of Bluetooth 4.0 specification and it is a specific feature subset designed to run on coin cells powered devices. It was designed with Internet Of Things [12] in mind, focusing on energy saving instead of aiming at high data transfer rates. Small and low cost devices often require an easy-to-implement protocol with very low energy consumption. The new protocol stack defines a new PHY layer, a new advertising mechanism, a new asynchronous connection-less MAC and a new asynchronous Client/Server architecture. BLE operates in the same ISM band as Bluetooth 1.0 [13], but it uses a new set of channels, new radio specifications and new protocol schemes to obtain a consumption reduction. One of the most interesting BLE features for smart city applications is the advertising capability, because it can be used to allow the interaction of BLEenabled devices such as mobile terminals to easily interact with the environment, thus implementing a physical presence/localization side to the applications. In the previous specifications of Bluetooth, the Master device was set to perform an active scan to find nearby Slave peripherals. Instead, BLE defines a Generic Access Profile (GAP) which controls the device connections and advertisments, that introduce two new roles for devices:

- Peripheral: small, low power devices like hearh rate monitor, proximity tag, etc

- Central: smartphone or tablet with more processing power, memory and and battery resources

A peripheral device can advertise itself by broadcasting periodic packets with a small amount of data (31 byte payload). The central device can scan to find the peripherals, and the responsivness for the system is achived reducing the channels used for advertising to 3. This little protocol modification allows the use of longer interval between advertising packets, thus guaranteing a fast detection by the central devices. In smart environment applications BLE can be used as a localization technology for both indoor and outdoor scenarios, in order to allow user interaction with the environment. Two different operation modes have been considered:

- Direct Mode: peripheral devices called anchors are located in the environment and the user devices (for example: smartphones) detect the anchors and take actions on a localization basis; this operation mode is similar to the iBeacon mode [14];

- Reverse Mode: the user device (i.e. personal tag) advertises itself with periodic message broadcasting, and the smart BLE devices placed in the environment perform a continuos scan to detect users proximity and take user-based actions.

In the first scenario anchor nodes can be very simple and cheap because they act like radio markers and when the user device (smartphone, tablet, ...) detects an anchor it can identify its unique ID and from this information it can determine its position from an anchor database server. A user device can take actions based on its position: e.g. it can prompt user to open a nearby door, it can turn the light on, etc. 


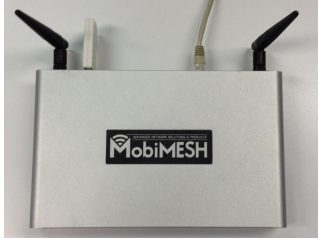

(a) AP

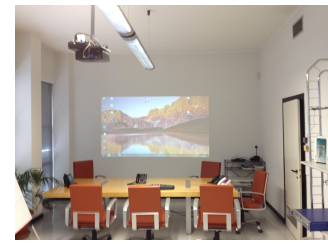

(b) Meeting room
Fig. 1. Testbed deployment

\section{TESTBED}

We have created a testbed to demonstrate how technologies integration opens the way to create a real life smart, energy efficient and user friendly environment with new applications. The specific focus of this testbed is to create a Smart Environment to help users automating interaction with local equipments; in particular the testbed has been deployed in an office environment, therefore it considers office devices and actions (personal desk pc, voip phone, office light, door opening), but the same model can be applied in Smart City and other Smart Environment scenarios. Figure 1 shows the WiFi-Zigbee gateways and one of the rooms of the office in which the testbed has been created.

The interaction with the user is achieved through a mobile APP; such APP detects the BLE anchors and it communicates its position through the WiFi connection to a central presence server that can control several Zigbee devices, that can take presence-based actions such as turning on and off a PC, a desk telephone, a light, etc. The Zigbee devices are connected to the server with some Zigbee gateways as described in section IV-C. We have deployed our solution in a 3 floor building shown in Figure 2 where the WiFi network provides both internet connectivity and acts as Zigbee/WiFi gateway, by forwarding information to the Zigbee network. We have created 3 separate Zigbee networks in order to measure temperature, illuminance and presence and to control lights, power sockets, etc. The 3 networks are controlled by a coordinator connected to a Zigbee/WiFi gateway that routes information towards a central server.

The WiFi/ZigBee gateways are implemented by equipping the Access Points with ZigBee interfaces, and they can be configured as to belog to one of the following families:

- Protocol Aware Gateway: the gateway device is equipped with a software component that translates packets from one protocol to a format compatible with the other protocol. This solution optimizes perfomances but it scarcely adaptive to the modifications that occur to each of the involved protocols;

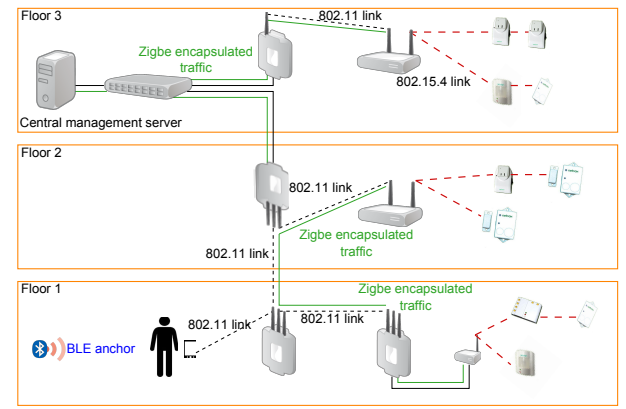

Fig. 2. Testbed network topology

- Protocol Unaware Gateway: the gateway device only incapsulates the packets coming from one side into packets of the other protocol, thus creating a transparent tunnel. This solution is slightly more bandwith consuming, but it is more flexible and easy to maintain, being end-to-end oriented.

In our testbed we have chosen the latter solution, because IEEE 802.11 has a larger bandwith than Zigbee, so encapsulation is not a big issue; moreover packet encapsulation provides a very flexible solution for future integration of other network technologies for smart sensors. The deployed system is also connected to the light control panel, to the projector, and to the power sockets of the users' PCs; we are also measuring the electricity consumption in order to measure how much energy can be saved. For example, we have considered the power consumption of a controlled meeting room (projector, PC used to show presentation). The energy consumption of each device in every state is reported in table I:

TABLE I

ENERGY CONSUMPTIONS

\begin{tabular}{|l|l|l|}
\hline Device & State & Consumption [mAh] \\
\hline \multirow{3}{*}{ Projector } & off & 0 \\
\cline { 2 - 3 } & standby & 286 \\
\cline { 2 - 3 } & on & 1400 \\
\hline \multirow{2}{*}{ PC } & off & 0 \\
\cline { 2 - 3 } & on & 200 \\
\hline
\end{tabular}

The meeting room has been monitored for 30 days to get the measurements of the power consumptions of the devices. It has turned out that the meeting room is used for an average of 4.3 hours per day (considering only working days), with a single meeting per day; during the meetings the PC has always been on and the projector has switched from the standby state to the active state using its own timeout based system. Moreover, after the meeting both the projector and the PC are left on, until 
the projector timeout intervened and changed the state to standby and then to off, while the PC was left always on. Table II shows the energy consumption obtained in such case.

TABLE II

ENERGY CONSUMPTION WITHOUT SMART ENVIRONMENT APPLICATION

\begin{tabular}{|l|l|l|l|l|}
\hline \multirow{2}{*}{ Device } & State & $\begin{array}{c}\text { Mean time } \\
\text { per day } \\
{[\text { hours] }}\end{array}$ & $\begin{array}{c}\text { Consumpt. } \\
{[\text { Wh] }}\end{array}$ & $\begin{array}{c}\text { Mean daily } \\
\text { consumpt. } \\
{[\text { Wh] }}\end{array}$ \\
\hline \multirow{3}{*}{ Projector } & off & 0 & 0 & \multirow{2}{*}{2563,924} \\
\cline { 2 - 4 } & standby & 19,7 & 1239,524 & \\
\cline { 2 - 4 } & on & 4,3 & 1324,4 & \multirow{2}{*}{1056} \\
\hline \multirow{2}{*}{ PC } & off & 0 & 0 & \\
\cline { 2 - 4 } & on & 24 & 1056 & $\mathbf{3 6 1 9 , 9 2 4}$ \\
\hline \multicolumn{3}{|l|}{} \\
\hline
\end{tabular}

With our Smart Environment system we can turn both devices off when they're not used, with a considerable cost saving. In fact the absence of people in the room, detected through the BLE-based localization system and infrared sensors, indicates that there is no need of the devices, so that they can be powered off; an hysteresis mechanism is also implemented, to avoid unwanted switching on and off in case of people temporarly leaving the room. Table III shows the power consumptions obtained in such a Smart Environment applicationcontrolled case.

TABLE III

ENERGY CONSUMPTION SMART ENVIRONMENT APPLICATION

\begin{tabular}{|l|l|l|l|l|}
\hline \multirow{2}{*}{ Device } & State & $\begin{array}{c}\text { Mean time } \\
\text { per day } \\
{[\text { hours] }}\end{array}$ & $\begin{array}{c}\text { Consumpt. } \\
{[\text { Wh] }}\end{array}$ & $\begin{array}{c}\text { Mean daily } \\
\text { consumpt. } \\
{[\text { Wh] }}\end{array}$ \\
\hline \multirow{3}{*}{ Projector } & off & 14 & 0 & \multirow{2}{*}{1683,044} \\
\cline { 2 - 4 } & standby & 5,7 & 358,644 & \\
\cline { 2 - 4 } & on & 4,3 & 1324,4 & \multirow{2}{*}{277,2} \\
\hline \multirow{2}{*}{ PC } & off & 17,7 & 0 & \\
\cline { 2 - 4 } & on & 6,3 & 277,2 & $\mathbf{1 9 6 0 , 2 4 4}$ \\
\hline \multicolumn{3}{|l|}{} \\
\hline
\end{tabular}

The energy saving is only one of the benefits gained from the Smart Environment, though being one of the easy measurable ones; the testbed will now be used to experiment more interactions and applications.

\section{CONCLUSION}

Smart Environments are an interesting area of technological development, in which different technologies are tied together to create value added services and applications. The success of such environments will be granted by the degree of interplay that will be achieved by the different technologies and by the capability of the applications to exploit the possibilities provided by the interaction of the components; therefore we have created a Smart Environment testbed which employs several technology components to create a Smart Environment where we measure some characterization parameters such as energy consumption reduction and to observer the advantages of our applications. More technologies will be added in the future, together with an easier interoperation platform in order to ease the interoperablity between technologies, and to better measure the benefits obtained by applications.

\section{ACKNOWLEDGMENT}

The work leading to these results has also received funding from the European Union's Seventh Framework Programme (FP7 MC-IAPP) under project no. grant agreement n [324515 MESH-WISE].

\section{REFERENCES}

[1] H. Schaffers, N. Komninos, M. Pallot, B. Trousse, M. Nilsson, and A. Oliveira, Smart cities and the future internet: Towards cooperation frameworks for open innovation. Springer Berlin Heidelberg, 2011.

[2] I. C. S. L. M. S. Committee et al., "Wireless lan medium access control (mac) and physical layer (phy) specifications," 1997.

[3] E. Perahia and R. Stacey, Next Generation Wireless LANs: $802.11 \mathrm{n}$ and $802.11 \mathrm{ac}$. Cambridge university press, 2013.

[4] L. Li, X. Hu, J. Huang, and K. He, "Research on the architecture of automatic meter reading in next generation network," in IEEE International Conference on Industrial Informatics (INDIN), 2008, pp. 92-97.

[5] E. DIN, “13757-4: 2005," Communication Systems for Meters and Remote Reading of Meters-Part, vol. 4, 2005.

[6] S. Spinsante, M. Pizzichini, M. Mencarelli, S. Squartini, and E. Gambi, "Evaluation of the wireless m-bus standard for future smart water grids," in IEEE International Wireless Communications and Mobile Computing Conference (IWCMC), 2013, pp. 1382-1387.

[7] S. Chalasani and J. M. Conrad, "A survey of energy harvesting sources for embedded systems," in IEEE Southeastcon, 2008, pp. 442-447.

[8] P. Baronti, P. Pillai, V. W. Chook, S. Chessa, A. Gotta, and Y. F. Hu, "Wireless sensor networks: A survey on the state of the art and the 802.15. 4 and zigbee standards," Computer communications, vol. 30, no. 7, pp. 1655-1695, 2007.

[9] L. S. Committee et al., "Part 15.4: wireless medium access control (mac) and physical layer (phy) specifications for lowrate wireless personal area networks (lr-wpans)," IEEE Computer Society, 2003.

[10] S. Bluetooth, "Specification of the bluetooth system core package version 4.0."

[11] R. Heydon and N. Hunn, "Bluetooth low energy," CSR Presentation, Bluetooth SIG https://www. bluetooth. org/DocMan/handlers/DownloadDoc. ashx, 2012.

[12] L. Atzori, A. Iera, and G. Morabito, "The internet of things: A survey," Computer networks, vol. 54, no. 15, pp. 2787-2805, 2010.

[13] S. Bluetooth, "Bluetooth specification version 1.1," [Accessed July 2014: Available ONLINE] HTTP: http://www. bluetooth. com, 2001.

[14] N. Newman, "Apple ibeacon technology briefing," Journal of Direct, Data and Digital Marketing Practice, vol. 15, no. 3, pp. 222-225, 2014. 